\title{
Commentary: Differential Humoral and Cellular Immunity Induced by Vaccination using Plasmid DNA and Protein Recombinant Expressing the NS3 Protein of Dengue Virus type 3
}

\author{
Angel Ramos-Ligonio', Aracely López-Monteon ${ }^{1 *}$
}

'LADISER Inmunología y Biología Molecular, Universidad Veracruzana, Orizaba, Veracruz, México

\section{Article Info}

\section{Article Notes}

Received: October 09, 2019

Accepted: November 27, 2019

\section{*Correspondence:}

Dr. Aracely López-Monteon, LADISER Inmunología y

Biología Molecular, Facultad de Ciencias Químicas

Universidad Veracruzana. Orizaba, Veracruz, México;

Telephone No: 52(272)7240120; Fax No: 52(272)7240120;

Email: aralopez@uv.mx.

C 2019 López-Monteo A. This article is distributed under the terms of the Creative Commons Attribution 4.0 International License.

\section{Keywords}

NS3 Protein

Dengue Virus

Vaccination

Immune Response

Immunomodulation
As it is known, dengue is a viral infection transmitted by mosquitoes (Aedes aegypti y A. albopictus) causing flu-like symptoms, sometimes the disease evolves into a deadly condition known as severe dengue (before dengue hemorrhagic fever). The incidence of dengue in last years has increased considerably, where more than half of the world's population has the risk of contracting this disease ${ }^{1}$. Dengue occurs in tropical and subtropical climates and preferably in urban and semi-urban areas, and in some countries of Asia and Latin America it is a serious cause of death in the child population, since today there is no specific treatment for this disease ${ }^{1}$. Dengue consists of four, closely related but antigenically, distinct viral serotypes (DENV1-4). In patients who have suffered from dengue, the immunity acquired against the serotype that caused the infection remains a lifetime. But, the immunity generated against other serotypes in a second infection represents a great risk, since this induced immunity increases in the patient the risk of suffering from severe dengue ${ }^{2}$. Due to the fact that Dengvaxia ${ }^{\circledR}$ (the only licensed dengue vaccine to date) increases the risk of severe illness among seronegative recipients, the WHO has recommended screening individuals for their serostatus prior to vaccination ${ }^{3}$, in addition, in a recent works it has been observed that by means of vaccination reinforcement in these patients, the antibody titer increased $^{4}$. According to the WHO, to determine the serological status of the subjects before vaccination and vaccinate only those who have been previously infected, could be a good alternative. In addition, the decision of implementing the strategy to determine the patient's serological status before vaccination requires careful evaluation, which must consider the sensitivity and specificity of the available evidence and local priorities, epidemiology, hospitalization rates, affordability of the vaccine and screening tests ${ }^{5}$. In this sense, scientists have focused on the manufacture of efficient biosensors for the detection of dengue virus with high precision, specificity, sensitivity, and simplicity ${ }^{6}$. These biosensors range from those involving colorimetric, improved surface, fluorometric, ELISA, PCR, electronic methods, the use of microfluids, to the use of smartphones are advanced strategies for the detection of various dengue serotypes $^{7}$. On the other hand, graphene shows great potential in the field of biomedicine where it hasn't been fully explored, and represents an opportunity for the development of new technologies with applicability in the administration of drugs or a development as biosensors, thanks to its large surface that can be used for the recognition of specific proteins of pathogens ${ }^{8}$. Finally, the detection 
of mRNA molecules in individual cells offer opportunities for research, medicine and molecular diagnosis. NanoFlare RNA detection probes have expanded the available possibilities of quantitative analysis at the level of mRNA in a single cell. The speed, sensitivity and versatility of this biosensor make it an ideal candidate for subsequent application and provides a promising diagnostic platform for early detection of dengue infection in high-riskareas with limited resources ${ }^{9,10}$.To date, all vaccines against dengue virus in formulation are tetravalent, with the objective of conferring protection of the four dengue serotypes. The controversy and suspension of the vaccination campaign was due to the low efficacy of the Dengvaxia ${ }^{\circledR}$ vaccine, it was because the vaccine increased the risk of severe dengue and hospitalization of people without prior exposure to the virus, this has caused skepticism about the safety of this vaccine. One of the most important risks is that the vaccine works differently when it comes to a population that hasn't been in contact with the virus (seronegative) who, when contracted for the first time, can develop a more severe clinical features. While tetravalent live-attenuated vaccines (LATVs), which mimic natural infection by inducing both cellular and humoral immune responses, are still currently favored, the development of a vaccine that provides a balanced immunity to all four DENV serotypes remains being a challenge $\mathrm{e}^{11}$.

Having a vaccine against the four strains of DENV which is safe, effective and affordable will be a breakthrough for disease control. This was one of the main triggers of the work of Hurtado-Melgoza et al. ${ }^{12}$. First, we would have to consider that DENV infection induces potent innate immune responses, which in turn shape the adaptive immune responses, including not only B cell humoral responses with antibody production but also cellular responses mediated by $\mathrm{CD} 4+$ and $\mathrm{CD} 8+\mathrm{T}$ cells ${ }^{13}$. The immunodominant epitopes recognized by CD4+ and CD8+ T cells have been mapped, with CD8+ T cells preferentially targeting NS proteins, such as NS3, NS4b and NS5; and $\mathrm{CD} 4+\mathrm{T}$ cell responses toward structural proteins and $\mathrm{NS} 1^{14}$ 15 , while the majority of T-cell epitopes are concentrated within the NS3 protein, the main target for CD4+ and CD8+ T-cell response $\mathrm{e}^{16,17}$.

In the work from Hurtado-Melgoza et al., we tested immunization with a DNA vaccine which encoding a protease domain of NS3 protein of serotype 3 (DEN3) and with the recombinant protein. The results showed that only splenocytes from mice immunized with the plasmid pcDNA3/NS3-DEN3 and stimulated with the homologous protein, induced a profile compatible with a protective response. In addition, only a weak antibody response against the NS3 was detected in the serum of DNA vaccinated animals. This response was of low magnitude, especially when compared to the recombinant protein vaccine in which antibody titers were 320 times higher. These results suggested that mice immunized with the gene coding for the NS3-DEN3 protein are primed to develop a Th1 response which provide protection against infection with dengue virus ${ }^{12}$.

Much of the positive results in the work of HurtadoMelgoza et al. was due to a possible manipulation of the system, from the point of view of the antigenic processing of a DNA vaccine which is very different from that of a proteinbased formulation ${ }^{18,19}$, and especially in the recombinant system where the absence of post-translational modifications affects the processing of proteins in a certain way, since the functionality or biological activity of glycoproteins is dependent on their interaction with their specific receptor. Dengue DNA vaccines have shown that the vaccine is safe and well tolerated, however, currently there are two challenges in this type of vaccines, 1 ) induce relatively weak immune responses in humans, and 2) It is difficult to identify the appropriate immunogenic peptides to arouse a good protective response ${ }^{20,21}$. In general, most studies emphasize the importance of the response of the $\mathrm{T}$ cells involved in the protection against dengue induced by DNA vaccines and above all maintain the immunogenicity of these applications. Recent reports have shown that electroporation of genetic material and its use on cells of immune importance as antigen presenting cells (macrophages and dendritic cells) represent new strategies for the development of DNA vaccines that enhance their immunological effect ${ }^{22,23}$.

In the case of NS3 protein, it is known that in its sequence there is a large number of epitopes for $\mathrm{T}$ cells and some studies have analyzed the use of these epitopes to be possible protective antigens against DENV infection, these assertions, sometimes lead us to think that most of these epitopes should be protective, however, the intrinsic characteristics of these epitopes, regardless of whether they are specific for $\mathrm{T}$ cells theirown or play another role during their antigenic presentation, in this sense, few studies have focused on the study of the immunomodulatory process of the NS3 protein in viral pathogenesis ${ }^{24}$. Unfortunately, due to the limitations that currently exist in the knowledge of vaccine immunity and to the implicit factors of the antigen, for some diseases it hasn't been easy to find good protection markers. Dengue virus NS3 protein is a multifunctional protein that plays an important role during viral replication ${ }^{25}$. Few are the studies where the NS3 protein is used as a vaccine candidate, recently it has been shown that the immunization of the NS1 and NS3 protein generates humoral and cellular immune responses providing a potential strategy for dengue vaccine design ${ }^{26}$. In this sense, the particles called VLPs (Virus-Like Particles) are being already implemented in dengue virus ${ }^{27}$. These particles can maintain the native conformation of 
the antigen and mimic the natural structure of the virus without containing viral genetic material and therefore not be infectious. The characteristics inherent to VLPs, very similar to those of viruses, they make them particularly effective in inducing a potent humoral immune response (antibody production). Also, they are capable of inducing cellular immune responses (proliferation of CD4+ and CD8+ lymphocytes), as well as activating antigen presenting cells (dendritic cells and macrophages). In addition, they can be used as vaccine vectors for the multimeric presentation of foreign epitopes (prophylactic vaccines), which are incorporated into the VLPs either by genetic fusion or by chemical conjugation, or for the presentation of own antigens (therapeutic vaccines) in applications aimed at the treatment of chronic diseases ${ }^{28,29}$.

In addition, that these particles can serve as an epitope presenting systemin conjugate with DNA vaccination could allow the production of more expected vaccines.

In conclusion, we need a dengue vaccine, however, we must be cautious and carefully analyze experimental studies before introducing public dengue vaccination programs. It is necessary to have vaccines of proven efficacy, effectiveness, safety, which confer long-term immunity, and also take into account the cost-effectiveness of vaccination, equity in access to this technology, and the financing possibilities for purchase of new vaccines. Let's remember that at stake is the health of those vaccinated and the credibility of vaccines.

\section{References}

1. WHO, 2019 Descriptive notes: dengue and severe dengue, 15 de abril de 2019, Available from: https://www.who.int/es/news-room/factsheets/detail/dengue-and-severe-dengue

2. Gubler DJ. The global pandemic of dengue/dengue haemorrhagic fever: current status and prospects for the future. Ann Acad Med Singapore. 1998; 27(2): 227-234.

3. Lam HM, Phuong HT, Thao Vy NH, et al. Serological inference of past primary and secondary dengue infection: implications for vaccination. J R Soc Interface. 2019; 16(156): 20190207. doi: 10.1098/rsif.2019.0207

4. Park J, Archuleta S, Oh MH, et al. Immunogenicity and safety of a dengue vaccine given as a booster in Singapore: a randomized Phase II, placebo-controlled trial evaluating its effects 5-6 years after completion of the primary series. Hum Vaccin Immunother. 2019; 29. doi: $10.1080 / 21645515.2019 .1661204$.

5. Pearson CAB, Abbas KM, Clifford S, et al. Serostatus testing and dengue vaccine cost-benefit thresholds. J R Soc Interface. 2019; 16(157): 20190234. doi: 10.1098/rsif.2019.0234.

6. Rodríguez RA, Pepper IL, Gerba CP. Application of PCR-based methods to assess the infectivity of enteric viruses in environmental samples. Appl Environ Microbiol. 2019; 75: 297-307.

7. Eivazzadeh -Keihan R, Pashazadeh-Panahi P, Mahmoudi T, et al. Dengue virus: a review on advances in detection and trends - from conventional methods to novel biosensors. Microchim Acta. 2019; 186: 329. https://doi.org/10.1007/s00604-019-3420-y

8. Askari E, Naghib SM, Seyfoori A, et al. Ultrasonic-assisted synthesis and in vitro biological assessments of a novel herceptin-stabilized graphene using three dimensional cell spheroid. Ultrason Sonochem. 2019; (58): 104615. doi: 10.1016/j.ultsonch.2019.104615.

9. Chenab KK, Eivazzadeh-Keihan R, Maleki A, et al. Biomedical applications of nanoflares: Targeted intracellular fluorescence probes. Nanomedicine NBM. 2019; (17): 342-358. doi: 10.1016/j. nano.2019.02.006. Epub 2019 Feb 28.

10. Yrad FM, Castañares J, Alocilja EC. Visual Detection of Dengue-1 RNA Using Gold Nanoparticle-Based Lateral Flow Biosensor. Diagnostics. 2019; (9)74. doi:10.3390/diagnostics9030074

11. Galula JU, Salem GM, Chang GJ, et al. Does structurally-mature dengue virion matter in vaccine preparation in post-Dengvaxia era? Hum Vaccin Immunother. 2019; (23): 1-9. doi: 10.1080/21645515.2019.1643676.

12. Hurtado-Melgoza ML, Ramos-Ligonio A, Álvarez-Rodríguez, et al. Differential humoral and cellular immunity induced by vaccination using plasmid DNA and protein recombinant expressing the NS3 protein of dengue virus type 3. J Biomed Sci. 2016; 23(1): 85.

13. Slon CJ, Mongkolsapaya J, Screaton G. The immune response against flaviviruses. Nat Immunol. 2018; (19): 1189-98. doi:10.1038/ s41590-018-0210-3.

14. Duangchinda T, Dejnirattisai W, Vasanawathana S, et al. Immunodominant T-cell responses to dengue virus NS3 are associated with DHF. Proc Natl Acad Sci U S A. 2010; (107): 16922-27. doi:10.1073/pnas.1010867107.

15. Grifoni A, Angelo MA, Lopez B, et al. Global assessment of dengue virus-specific CD4 T cell responses in dengue-endemic areas. Front Immunol. 2017; (8): 1309. doi:10.3389/fimmu.2017.01309.

16. Mathew A, Rothman AL. Understanding the contribution of cellular immunity to dengue disease pathogenesis. Immunological Reviews. 2008; (255): 300-313.

17. Appanna R, Tan LH, See LLC, et al. Cross-reactive T-cell responses to the nonstructural regions of dengue viruses among dengue fever and dengue hemorrhagic fever patients in Malaysia. Clin Vaccine Immunol. 2007; (8): 969-977.

18. Srivastava IK, Liu MA. Gene vaccines. Ann Intern Med. 2003; (138): 550-559.

19. Shedlock DJ, Weiner DB. DNA vaccination: antigen presentation and the induction of immunity. J Leukoc Biol. 2000; (68): 793-806.

20. Williams M, Ewing D, Blevins M, et al. Enhanced immunogenicity and protective efficacy of a tetravalent dengue DNA vaccine using electroporation and intradermal delivery. Vaccine. 2019; 37(32): 4444-4453. doi: 10.1016/j.vaccine.2019.06.083

21. Pinto PBA, Assis ML, Vallochi AL, et al. T Cell Responses Induced by DNA Vaccines Based on the DENV2 E and NS1 Proteins in Mice: Importance in Protection and Immunodominant Epitope Identification. Front Immunol. 2019; (10): 1522. doi: 10.3389/fimmu.2019.01522

22. Sheng Z, Chen H, Feng K, et al. Electroporation-Mediated Immunization of a Candidate DNA Vaccine Expressing Dengue Virus Serotype 4 prM-E Antigen Confers Long-Term Protection in Mice. Virol Sin. 2019; (1): 88-96. doi: 10.1007/s12250-019-00090-8.

23. Zaneti AB, Yamamoto MM, Sulczewski FB, et al. Dendritic Cell Targeting Using a DNA Vaccine Induces Specific Antibodies and CD4+ T Cells to the Dengue Virus Envelope Protein Domain III. Front Immunol. 2019; (10): 59. doi: 10.3389/fimmu.2019.00059.

24. Rothman AL. Immunity to dengue virus: a tale of original antigenic in and tropical cytokine storms. Nat Rev Immunol. 2011; (11): 532-543.

25. Gebhard LG, Iglesias NG, Byk LA, et al. 2016. A proline rich N-terminal region of the dengue virus NS3 is crucial for infectious particle production. J Virol. 2016; (90): 5451-5461. doi:10.1128/JVI.00206-16.

26. Kao YS, Yu CY, Huang HJ, et al. Combination of Modified NS1 and NS3 
as a Novel Vaccine Strategy against Dengue Virus Infection. J Immunol. 2019; 203(7): 1909-1917. doi: 10.4049/jimmunol.1900136.

27. Urakami A, Ngwe Tun MM, Moi ML, et al. An Envelope-Modified Tetravalent Dengue Virus-Like-Particle Vaccine Has Implications for Flavivirus Vaccine Design. J Virol. 2017; 91(23). pii: e01181-17. doi: 10.1128/JVI.01181-17.
28. Changotra H, Vij A. Rotavirus virus-like particles (RV-VLPs) vaccines: An update. Rev Med Virol. 2017; 27(6). doi: 10.1002/rmv.1954

29. Roldão A, Mellado MC, Castilho LR, et al. Virus-like particles in vaccine development. Expert Rev Vaccines. 2010; 9(10): 1149-76. doi: 10.1586/erv.10.115. 